\title{
Catheter Ablation with Radiofrequency Energy: Biophysical Aspects and Clinical Applications
}

\author{
STEVEN J. KALBFLEISCH, M.D., and JONATHAN J. LANGBERG, M.D., F.A.C.C. \\ From the Division of Cardiology, Department of Internal Medicine, University of Michigan Medical Center, \\ Ann Arbor, Michigan
}

\begin{abstract}
Radiofrequency Catheter Ablation. Radiofrequency catheter ablation techniques are becoming increasingly accepted as the therapy of choice for selected patients with symptomatic arrhythmias. The ability to titrate the power output using radiofrequency current has allowed these ablative techniques to be applied safely in a variety of arrhythmias. In many institutions, radiofrequency catheter ablation has now become standard therapy for controlling medically refractory atrial arrhythmias using atrioventricular $(\mathrm{AV})$ junction ablation and for curing $\mathrm{AV}$ nodal reentrant tachycardia and supraventricular tachycardia due to accessory AV connections. This technology is also being used to treat some forms of ventricular tachycardia such as bundle branch reentry ventricular tachycardia, ventricular tachycardia in structurally normal hearts, and with limited success in patients with ventricular tachycardia and coronary artery disease. Advancements in catheter design and energy delivery systems may further expand the use of this form of therapy. (J Cardiovasc Electrophysiol, Vol. 3, pp. 173-186, April 1992)
\end{abstract}

radiofrequency current, catheter ablation, supraventricular tachycardia, ventricular tachycardia

\section{Introduction}

Percutaneous transvenous catheter ablation of myocardial tissue has been used since the early $1980 \mathrm{~s}$ for treatment of supraventricular and ventricular arrhythmias. ${ }^{1-3}$ High-voltage direct current pulses from a standard defibrillator were used as the first ablative energy source. Although effective, the use of direct current shocks is associated with significant limitations, including the need for general anesthesia, and the risk of serious barotraumatic side effects. ${ }^{4}$

Energy produced by alternating current in the radiofrequency range has been used in electrosurgery for over 50 years. Radiofrequency current applied via needles has been used to create discrete lesions in the central and peripheral nervous systems $\mathrm{s}^{5,6}$ and for adjuvant thermotherapy of malignancies. ${ }^{7}$

Studies in animals suggested that percutaneous radiofrequency catheter ablation could be used

Address for correspondence: Jonathan J. Langberg, M.D., University of Michigan Medical Center, Division of Cardiology, 1500 East Medical Center Drive, BF222, Ann Arbor, MI 48109. Fax: 313936-7641.

Manuscript received 15 October 1991; Accepted for publication 11 December 1991. safely to create discrete lesions in the atria, ventricles, and atrioventricular (AV) junction. ${ }^{8-10}$ Subsequent reports as early as 1987 showed that radiofrequency ablation was safe and effective in humans and this has been corroborated in several recent larger series. ${ }^{11-15}$ In a number of institutions, radiofrequency catheter ablation has now supplanted surgical ablation for definitive cure of the Wolff-Parkinson-White syndrome and paroxysmal supraventricular tachycardia due to AV nodal reentry, and will likely become the standard of care for the nonpharmacologic management of these arrhythmias. ${ }^{13-15}$

\section{The Biophysics of Radiofrequency Lesion Formation}

Radiofrequency refers to alternating current in the range of 0.1 to $100 \mathrm{MHz}$. When used for ablation of cardiac arrhythmias, continuous, unmodulated sine wave outputs in the range of 100 to $750 \mathrm{kHz}$ are used. This is distinctly different from the waveform used for electrosurgical cutting and coagulation, where high-voltage, short duration pulses with a long duty cycle are designed to promote arcing or coagulum formation (Fig. 1). 

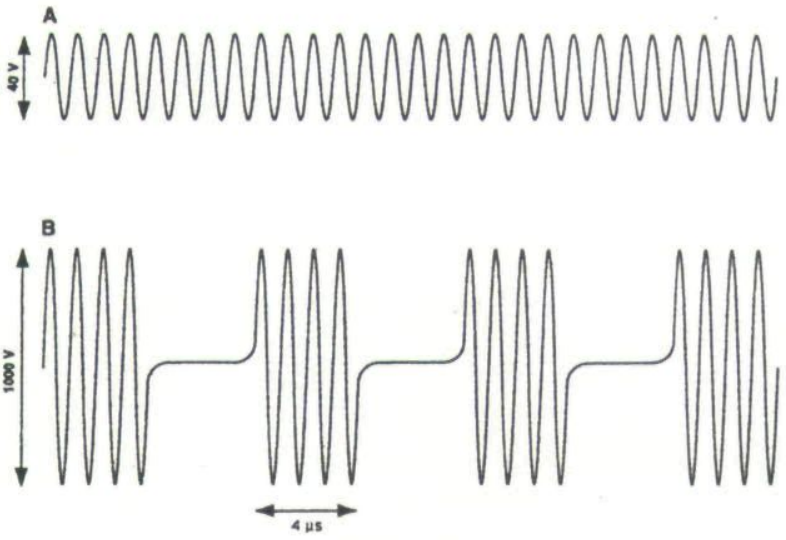

Figure 1. Waveforms used for radiofrequency cardiac catheter ablation $(A)$ and for electrosurgery $(B)$. Energy used for cardiac ablation is applied as a continuous, unmodulated sine wave of approximately $500 \mathrm{kHz}$. Outputs are typically in the range of 40-60 volts (V). In contrast, cutting and coagulation waveforms from electrosurgical units are much higher voltages and applied as a series of short pulses.

The mechanism of lesion formation by radiofrequency energy is via thermal injury to the tissue. ${ }^{6}$ The heating of cells drives water from them, leading to desiccation and coagulative necrosis, which occurs at a temperature of approximately $48^{\circ} \mathrm{C} .1^{16,17}$ During catheter ablation, current flows from the catheter electrode tip to the tissue, resulting in acceleration of ions and resistive heating of the electrode-tissue interface. Since the power density falls off exponentially as a function of distance from the electrode, only a small rim of tissue around the catheter tip is heated directly by the current. The majority of thermal injury is mediated by conduction of heat away from the area of electrode contact into the surrounding tissue (Fig. 2). Therefore, the temperature gradient seen during radiofrequency ablation is accurately modeled as a heat source with a diameter only slightly larger than the ablating electrode. Provided the duration of energy application is adequate to achieve a steady state, lesion size can be predicted by electrode diameter and tip temperature. ${ }^{17}$ Lesion size is limited by the fact that tip temperatures above $100^{\circ} \mathrm{C}$ result in coagulum formation that coats the ablating electrode. This insulates the electrode, causing an abrupt rise in impedance that limits current flow and prevents further lesion expansion. $5,17,18$

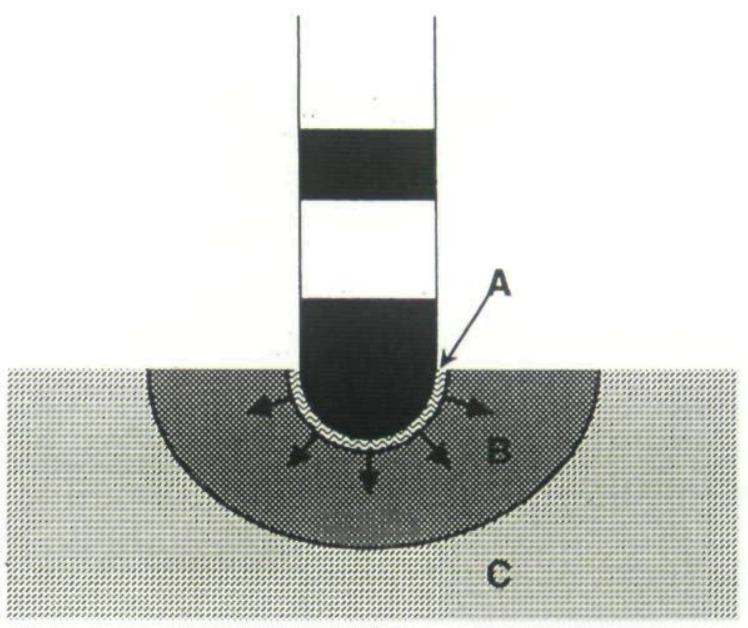

Figure 2. Schematic diagram of radiofrequency current delivery from the tip of a bipolar ablation catheter and its effect on adjacent myocardium. The thin rim of tissue around the catheter tip is the zone of resistive heating $(A)$. This is the only area of the myocardium that is directly affected by the current flow. The surrounding zone of necrosis $(B)$ is created by thermal injury by heat conducted from the zone of resistive heating. The arrows indicate flow of heat away from the electrode-tissue interface and into the neighboring myocardium. The portion of myocardium unaffected by the radiofrequency current and secondary thermal effects is labeled $C$.

\section{Radiofrequency Energy Delivery Parameters}

The magnitude of high-voltage shocks used for catheter ablation is usually estimated by the energy stored in the defibrillator capacitors. Since the duration of current flow is extremely brief, there is some correlation between stored energy, peak voltage, and biological effects.

The mechanism of radiofrequency lesion formation is completely different. With onset of current flow, the temperature at the electrode-tissue interface increases over a period of $10-40$ seconds until the energy applied via the ablation catheter equals that lost through conduction and convection of heat away from the site. ${ }^{19}$ Once a steady-state temperature gradient has been achieved, there is no further lesion expansion. Based on this model, total delivered energy (power $\mathrm{x}$ duration) would be expected to correlate with lesion volume only during the early growth phase, but not with applications of longer duration. ${ }^{19}$

Studies of radiofrequency lesion formation in vitro show better a correlation between applied power, duration of application, total energy, and resultant lesion size than comparable studies in 
vivo. ${ }^{19-21}$ This most likely reflects the multitude of variables that can influence temperature at the electrode surface, including contact pressure, current density, and convective heat loss via intracavitary blood flow. ${ }^{20}$

\section{Radiofrequency Power Supply Design}

Several characteristics of a lesion generation system are necessary for safe and effective operation. Output that can be varied between 10 and 40 watts (W) is required. As in all medical electronics, the device must be electrically isolated to prevent the possibility of leakage current producing arrhythmias or injury. Output parameters (voltage, current, or power) must be monitored to allow detection of an impedance rise due to coagulum formation on the ablating electrode. Continued energy application after impedance rise may result in charring and excessive heating with the possibility of catheter disruption. ${ }^{22}$ Electrophysiologic recording systems have widely varying susceptibility to interference during ablation. Adequate filtering must be used to allow continuous monitoring of the surface electrocardiogram (ECG) during energy application.

\section{Radiofrequency Ablation Catheter Design}

A number of studies have examined the relationship between electrode configuration and radiofrequency lesion characteristics. Active fixation electrodes result in bigger lesions in vitro than do similar-sized passive contact electrodes, ${ }^{23}$ presumably because of the larger surface area in direct contact with the myocardium.

The effect of electrode size on lesion volume in vivo was examined in our laboratory. ${ }^{24}$ The results of radiofrequency application using a standard 6 French electrode catheter were compared to those using catheters modified with longer distal electrodes. Increasing electrode length from 2 to $4 \mathrm{~mm}$ more than doubled mean lesion volume from 143 to $326 \mathrm{~mm}^{3}$ at the same applied power. Increasing electrode length beyond $4 \mathrm{~mm}$ produced progressively smaller lesions, either because of a lower power density or as the result of contact between the proximal portion of the electrode and the blood pool.

Other studies have shown that cooling of the distal electrode with saline infused via a central lumen may delay or prevent the occurrence of coagulum formation and impedance rise, allowing larger maximal lesion size to be achieved. ${ }^{25,26}$

\section{Clinical Application of Radiofrequency Catheter Ablation}

\section{Ablation of the Atrioventricular Junction}

Huang and co-workers ${ }^{27}$ described the use of radiofrequency catheter ablation for production of complete AV block in experimental animals. They were able to achieve complete AV block in over $80 \%$ of the animals. The safety and efficacy of this technique has been corroborated by other investigators as well. ${ }^{8}$

Early experience in patients undergoing radiofrequency catheter ablation of the $\mathrm{AV}$ junction was characterized by modest success rates of approximately $50 \%-60 \%$ without significant complications or mortality. ${ }^{28,29}$ Jackman et al. ${ }^{12}$ achieved a higher success rate with conventional electrode catheters, but required an increased number of energy applications.

The introduction of large-tipped (3-4 $\mathrm{mm}$ in length) steerable ablation catheters has dramatically improved success rates from approximately $60 \%$ to greater than $90 \%$, decreased the incidence of impedance rise due to coagulum formation, and decreased the number of applications required to achieve $\mathrm{AV}$ block. ${ }^{12,30}$ Using the large tip electrode, Langberg et al. ${ }^{30}$ were able to achieve complete AV block in 12 of 13 patients using a mean of $18 \pm 6 \mathrm{~W}$ of power and $7 \pm 5$ applications. Jackman et al. also demonstrated the superiority of the large tip electrode for AV junction ablation. With the use of the large tip electrode they achieved complete AV block in 10 of 10 patients versus 6 of 7 patients with the small tip electrode. Compared to the small tip electrode, the large tip achieved complete AV block with fewer radiofrequency applications $(4.7 \pm 4.6 \mathrm{vs}$ $42 \pm 22$ ), and allowed delivery of greater power $(33 \pm 13.0 \mathrm{~W}$ vs $10.2 \pm 0.6 \mathrm{~W})$ without coagulum formation. ${ }^{12}$ Currently in our laboratory, we use $30-35 \mathrm{~W}$ of power delivered for 30-60 seconds when complete AV block is desired.

Patients who fail radiofrequency ablation may still be successfully treated with high-voltage, direct current shock ablation. An innovative technique to produce block using radiofrequency lesions applied to the mitral annulus has recently been described. ${ }^{31}$ Sousa et al. found this to be successful in 8 of 8 patients who had failed attempts at ablation of the AV junction from the right side. The ablation catheter is advanced into the femoral artery and then passed retrogradely 
across the aortic valve to the left ventricle where it is positioned against the septal aspect of the mitral annulus at a site with the largest His electrogram amplitude. Radiofrequency current is then applied at an output of 20-36 W for up to 60 seconds. Using this technique when ablation from the right side is ineffective has obviated the need for direct current shock ablation of the AV junction in our laboratory.

In order to successfully interrupt conduction with radiofrequency applied to the tricuspid annulus, the ablation catheter must be positioned more proximally than during a diagnostic electrophysiologic test. An "ideal" site is one where an atrial:ventricular electrogram ratio is > 1 and a large $(>150 \mu \mathrm{V})$ His electrogram can be recorded. Application to the site of the largest His electrogram will often be too distal, resulting in right bundle block rather than complete AV block. Several studies suggest that the occurrence of an accelerated junctional rhythm during energy application is a sensitive but nonspecific indication of an effective site. ${ }^{30,32}$ Our approach is to discontinue radiofrequency energy application and reposition the catheter if no junctional ectopy or change in conduction is observed after 20 seconds.

\section{Radiofrequency Catheter Ablation for Treatment of AV Nodal Reentrant Tachycardia}

Over the last decade, surgical ablation has been shown to be a safe and efficacious therapy for drug refractory AV nodal reentry. ${ }^{33,34}$ Intraoperatively, either cryothermia or electrophysiologically-guided sharp dissection is used around the proximal AV junction. These techniques have been able to eliminate reentry while still preserving antegrade conduction. Initial experience with radiofrequency ablation to produce variable degrees of AV block in animals suggested that the safety and titratibility of this energy source would make it well suited for treatment of AV nodal reentry. ${ }^{35-37}$ Early reports on small series of patients confirmed that radiofrequency catheter ablation could be used to eliminate reentry with preservation of conduction. ${ }^{38-41}$

Two different approaches to radiofrequency catheter modification of $\mathrm{AV}$ nodal reentrant tachycardia are now being used. The first technique developed was one where lesions are applied slightly anterior and proximal to the site of the maximal His electrogram ${ }^{13,14}$ (Fig. 3).
Target sites typically have an atrial:ventricular electrogram ratio $>1$ and a small $(<100 \mu \mathrm{V})$ His electrogram. Radiofrequency energy is applied until there is approximately $50 \%$ prolongation of the PR interval or a nonconducted $\mathrm{P}$ wave is observed. Junctional tachycardia often occurs during ablation and atrial overdrive pacing may be required to allow continuous assessment of conduction. After ablation, there is prolongation of the atrial (A) to His bundle $(\mathrm{H})$ interval, usually without a change in the antegrade Wenckebach cycle length or effective refractory period. Retrograde conduction is eliminated in approximately $40 \%$ of patients. The remainder have marked attenuation of retrograde conduction, with prolongation of the retrograde Wenckebach cycle length and ventriculoatrial conduction time (Fig. 4). AV nodal function curves after ablation appear similar to the slow pathway portion of the curve before the procedure. All these changes post-ablation are consistent with selective ablation of the fast AV nodal pathway. ${ }^{12,13,42,43}$

Using this approach, success rates of $82 \%$ and $95 \%$ were achieved in two large series. However, inadvertent complete AV block occurred in $8 \%$ and $2 \%$ of patients, respectively. ${ }^{13,14}$ We have

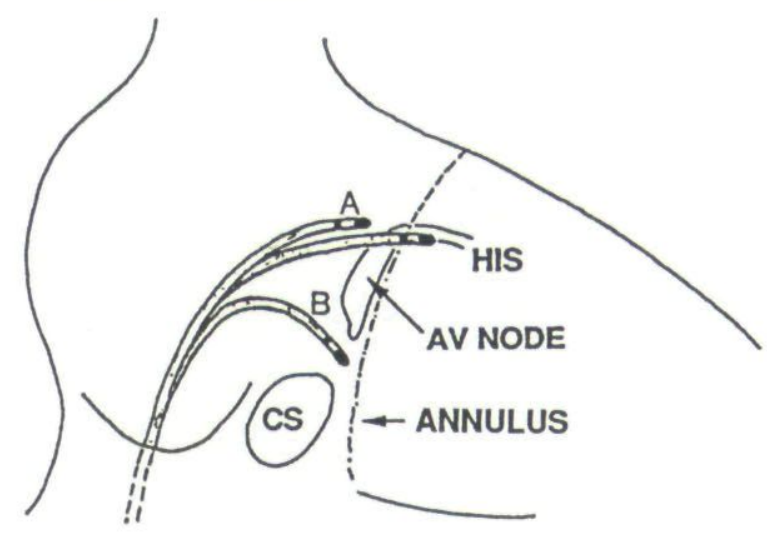

Figure 3. Ablation catheter locations for radiofrequency modification of the atrioventricular $(A V)$ node. " $A$ " shows the position of the ablation catheter for the fast pathway approach. This catheter is initially positioned to record a large His deflection (showed by the unlabeled catheter) and is withdrawn to a site with a relatively large atrial electrogram $(\geq 0.5 \mathrm{mV})$, small His deflection $(\leq 0.04 \mathrm{mV})$, and atrial:ventricular electrogram ratio $\geq 1$. At this anterior location, radiofrequency current is then applied initially at a low-power output (10 watts) and increased until the desired effect is seen. " $B$ " shows the position for ablation of the slow pathway. This is located posteriorly and is usually in close proximity to the coronary sinus (CS) ostium. 
recently modified our technique in an attempt to decrease the risk of high-grade AV block. Initially, ablation of the target site began with relatively high power ( $>15 \mathrm{~W}$ ) and the duration of the application was used to titrate the electrophysiologic effect. We now begin radiofrequency application at each new site at a power of $10 \mathrm{~W}$ for up to 20 seconds. If no effect is seen, power is increased in increments of $5 \mathrm{~W}$ with each 20-second test application until the desired effect is achieved or up to a maximum of $30 \mathrm{~W}$. By titration of applied power and duration, we have avoided inadvertent AV block in a consecutive series of 32 patients.

An exciting alternative approach to radiofrequency modification of $\mathrm{AV}$ nodal reentrant tachycardia has been developed using lesions applied near the ostium of the coronary sinus aimed at destroying the slow AV nodal pathway. This "posterior approach" is attractive because ablative injury occurs distant from the distal AV junction and His bundle, and it appears that the risk of high-grade AV block may be less. The target site for this technique has an atrial: ventricular electrogram ratio of approximately 1:2. A high-frequency deflection after the offset of the atrial electrogram also appears to be a sensitive but nonspecific indicator of an effective site (Fig. 5). As with ablative lesions made anteriorly, radiofrequency energy applied to an effective site posteriorly is often associated with junctional tachycardia. Lesions made in this area appear to selectively affect slow AV nodal pathway function. After ablation, there is no change in the A-H interval or in retrograde conduction or refractoriness. There may be slight prolongation of the antegrade Wenckebach cycle length and effective refractory period.

Roman et al. ${ }^{44}$ in a preliminary report, described elimination of slow pathway function in 12 of 13 patients. In an ongoing, prospective comparison of the two techniques of AV nodal modification, our success rate with the posterior approach is approximately $60 \%$. Larger studies are needed to define the safety and efficacy of the posterior approach. Since elimination of slow pathway function preserves a normal PR interval and may obviate the risk of high-grade block, the posterior approach may become the technique of choice, with crossover to the anterior approach only if the slow pathway cannot be successfully ablated.

The ability to selectively damage different parts of the reentrant loop has greatly contributed

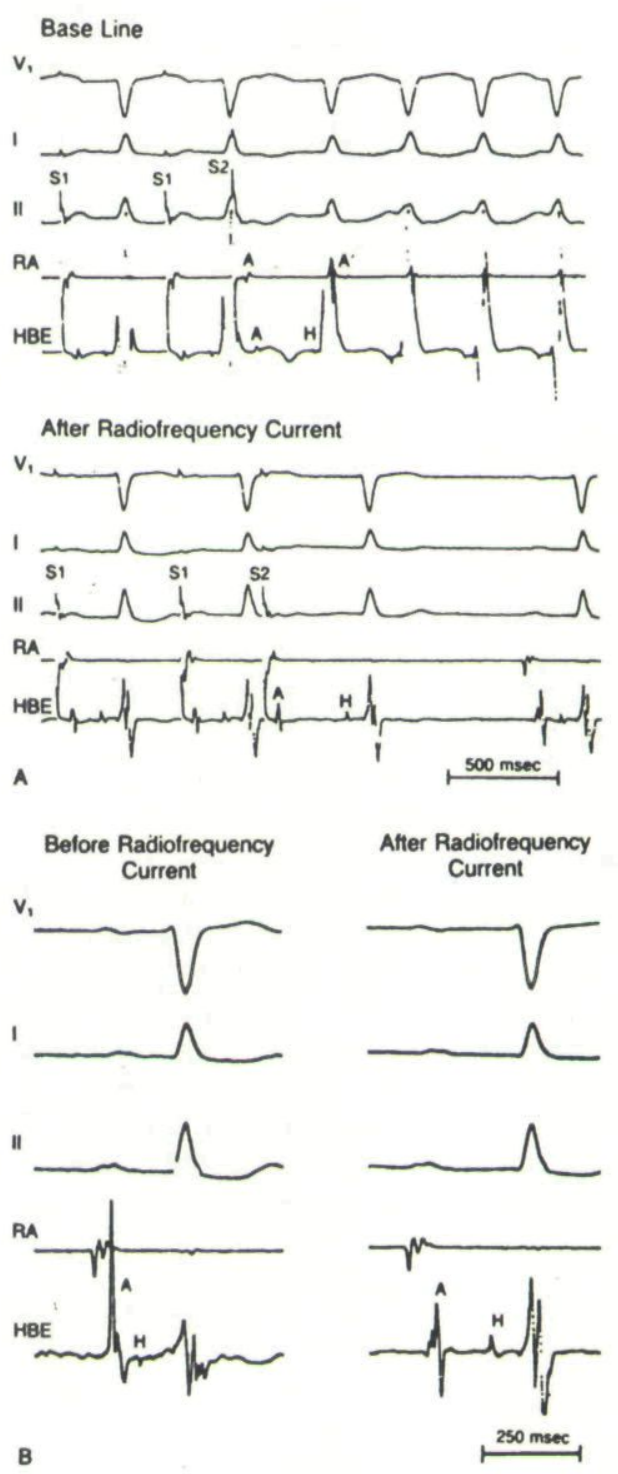

Figure 4. Typical atrioventricular (AV) nodal reentrant tachycardia eliminated by radiofrequency current. In the baseline state (A), typical $A V$ nodal reentry was induced by atrial extrastimulation. The tracings from leads $V_{1}, I$, and II are shown along with a recording from the right atrium (RA) and the His-bundle electrogram (HBE). An atrial extrastimulus (S2) introduced after several cycles of pacing (S1) at a rate of 120 beats/min resulted in prolongation of the atrial $(A)$-His bundle $(H)$ interval and an echo $\left(A^{\prime}\right)$, followed by sustained tachycardia at a rate of 175 beats/ min. After application of radiofrequency current through a catheter positioned near the $A V$ node, $A V$ nodal reentrant tachycardia could no longer be induced. Panel B shows the site at which radiofrequency current was delivered in the His-bundle electrogram. The amplitudes of the atrial, His, and ventricular $(V)$ electrograms were $0.6,0.02$, and 0.25 $m V$, respectively. After 16 watts of radiofrequency current delivered for 60 seconds, the A-H bundle interval was 170 msec as compared with $90 \mathrm{msec}$ at baseline. (Reproduced with permission from the New England Journal of Medicine. ${ }^{14}$ ) 


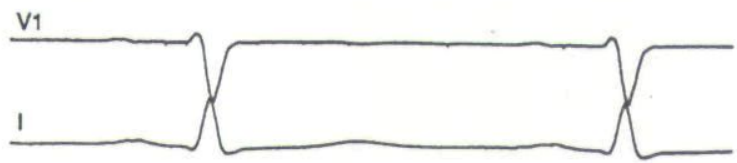

II

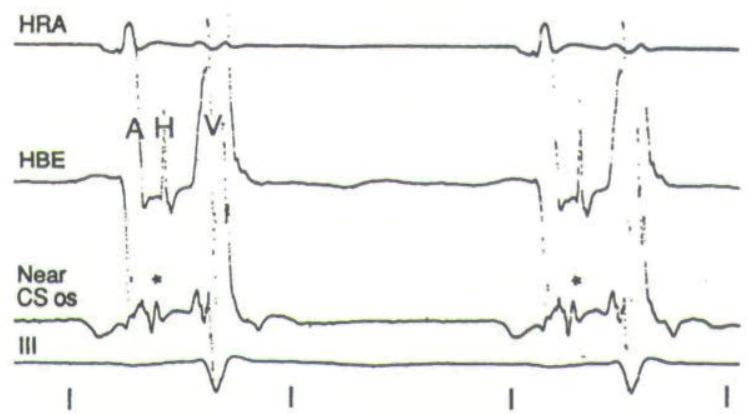

Figure 5. Site of $A V$ nodal modification aimed at elimination of the slow pathway. Shown are surface electrocardiographic leads VI, I, II, intracardiac recordings from the high right atrium (HRA), His-bundle electrogram (HBE) with the atrial $(A)$, His $(H)$, and ventricular $(V)$ deflections marked, recordings from the distal electrode pair of the ablation catheter placed near the coronary sinus ostium (CS os), and surface lead III. The time lines are $500 \mathrm{msec}$. The ablation catheter recording shows a fractionated atrial electrogram with a fairly discrete electrical potential $\left(^{*}\right)$ at the terminal portion of the atrial electrogram.

to our understanding of the pathogenesis of typical AV nodal reentrant tachycardia. It is now clear that two anatomically distinct inputs to the distal AV node form the antegrade and retrograde limbs of the circuit. The ability to selectively eliminate slow or fast pathway conduction at widely disparate sites argues strongly against functional longitudinal dissociation within the node itself as the mechanism of reentry.

We have observed new "atypical" AV nodal reentry after successful fast pathway AV nodal modification. ${ }^{45}$ This arrhythmia occurred in $11 \%$ of patients treated with anterior lesions and was characterized by an RP > PR interval and was induced exclusively with ventricular pacing during isoproterenol infusion. This arrhythmia most likely resulted from an increase in fast pathway refractoriness without complete elimination of fast pathway function. Although none of the patients were treated, only one had an episode of spontaneous atypical tachycardia during follow-up.

\section{Radiofrequency Ablation of Accessory Pathways}

The use of radiofrequency catheter ablation to destroy extranodal accessory AV connections is one of the most significant recent advances in arrhythmia management (Fig. 6). In many institutions, catheter ablation has largely replaced arrhythmia surgery for definitive treatment of patients with the Wolff-Parkinson-White syndrome. ${ }^{14,15}$

Techniques used to position the ablation catheter vary according to the location of the accessory pathway. Left-sided pathways are usually ablated using a catheter in the left

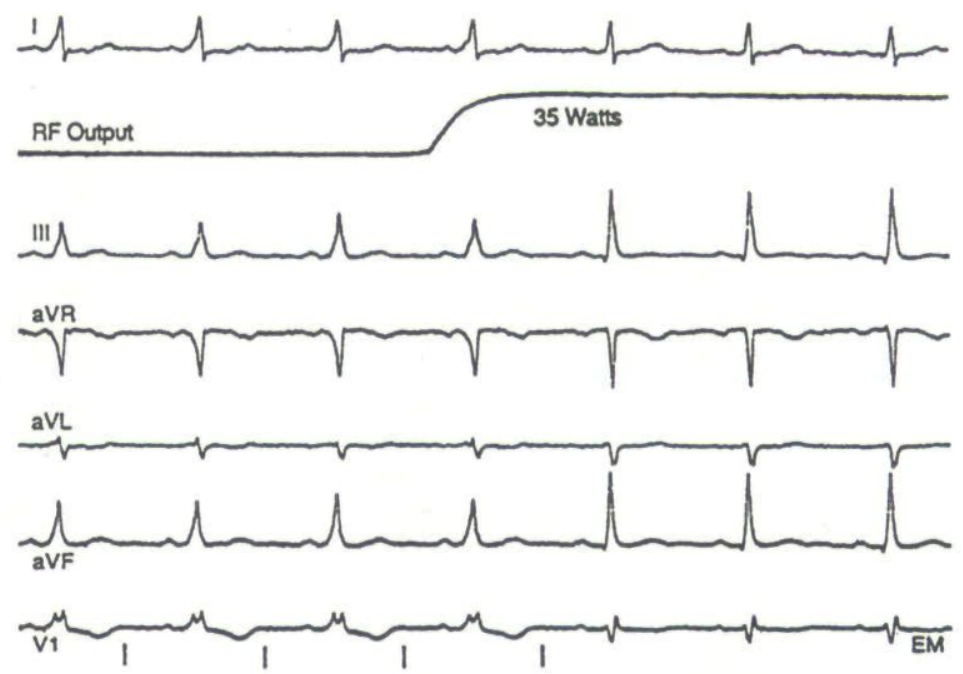

Figure 6. Surface electrocardiogram recording during ablation of a left anterolateral accessory pathway. Shown are surface lead I, radiofrequency (RF) power output, surface leads III, aVR, aVL, aVF, and VI. Time lines are 1 second. Application of $R F$ current at a power output of 35 watts resulted in a rapid loss (within 1 beat) of pre-excitation. 
ventricle. The electrode catheter is introduced into the femoral artery and passed retrograde across the aortic valve into the left ventricle. It is then manipulated between the mitral leaflet and ventricular endocardium and advanced to the level of the annulus where radiofrequency current is applied (Fig. 7). The ablation catheter can often be placed in the left atrium using this approach and may then be withdrawn to the level of the annulus with lesions applied on the atrial surface at the base of the mitral leaflet.

A transseptal approach has also been used to ablate left-sided accessory pathways with radiofrequency energy. ${ }^{46}$ After transseptal puncture, the electrode catheter is introduced into the left atrium through the transseptal sheath. Manipulation of the sheath and the steerable electrode catheter facilitates positioning and stability on the atrial side of the mitral annulus. At our institution, this approach is reserved for patients who have failed attempts using the retrograde aortic approach.

Femoral venous access is most often used for ablation of septal and right free-wall accessory pathways. The catheter is positioned on the atrial side of the tricuspid annulus. Occasionally, venous access from above (either internal jugular or subclavian) may be helpful for catheter placement on the tricuspid annulus. This is particularly useful for pathways located in the anterior and anterolateral areas. Although posteroseptal accessory pathways are most often ablated with lesions applied to the endocardium from the right side, some are interrupted with lesions made at or just inside the ostium of the coronary sinus or from the left ventricle using the retrograde aortic approach. 47

A study by Wang et al. ${ }^{48}$ evaluated the energy requirements necessary for successful ablation of accessory pathways. They found that at a power output of $30 \mathrm{~W}$, a pulse duration of $\geq 12$ seconds was required to create block lasting longer than 30 minutes in the accessory pathway. Another important observation from this study was that if block did not occur within the first 4 seconds of radiofrequency current application, it was unlikely to result in permanent block. At our institution we usually deliver radiofrequency current at a power output of $30-36 \mathrm{~W}$ for a 5 - to 10 -second test application. If the test application is unsuccessful, the catheter is repositioned. If the test application results in loss of accessory pathway conduction, then the radiofrequency current is then delivered for an additional 30-60 seconds.

Bipolar endocardial electrograms are most often used to localize accessory pathways. The ratio of atrial:ventricular electrogram amplitudes

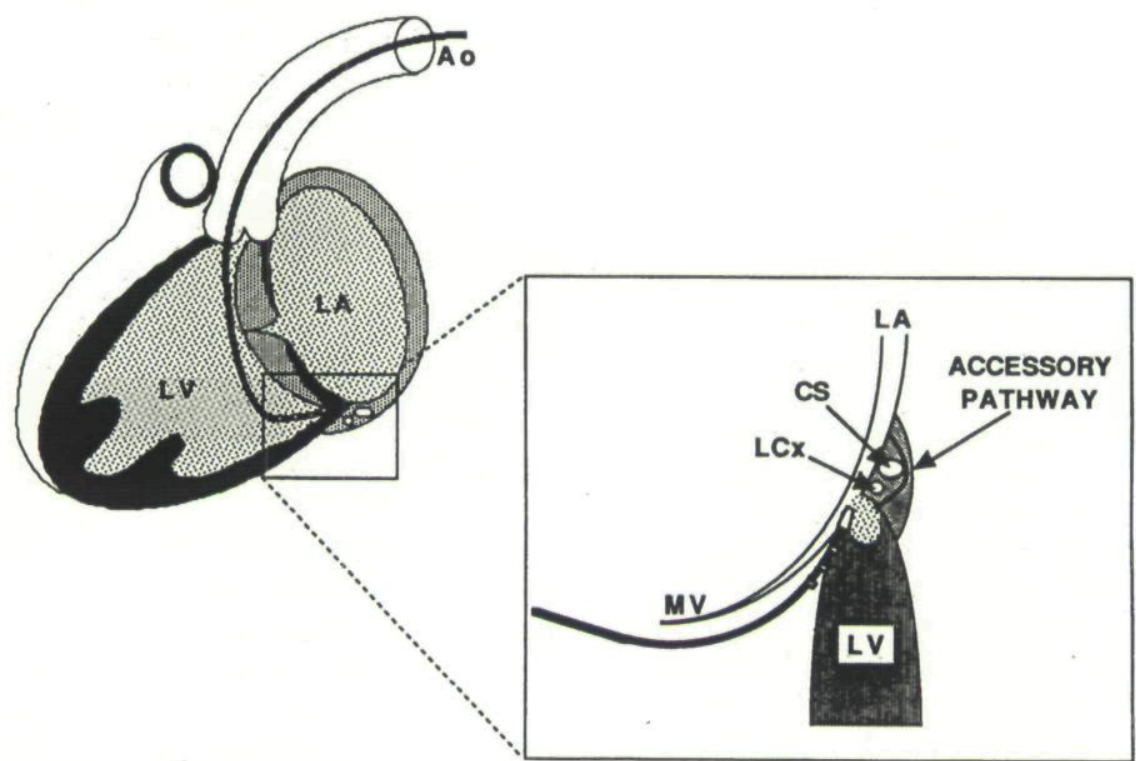

Figure 7. Ablation of a left-sided accessory atrioventricular (AV) connection using the retrograde aortic approach. The majority of left-sided accessory pathways can be successfully ablated by advancing the ablation catheter retrograde across the aortic valve into the left ventricle $(L V)$ and against the mitral annulus. The blown-up portion of the figure shows the position of the ablation catheter between the mitral valve $(M V)$ and left ventricular endocardium. The locations of the left circumflex artery $(L C x)$, coronary sinus $(C S)$, and accessory pathway traversing the $A V$ groove are also shown. LA = left atrium. 
is used to estimate electrode placement relative to the annulus. We have recently evaluated ablation site electrograms in an attempt to identify predictors of efficacy. ${ }^{49}$ During ablation of both concealed and manifest accessory pathways, electrogram stability (defined as $<10 \%$ change in amplitude with successive beats) was found to be an important determinant of success. Radiofrequency current applied at unstable sites had less than a $10 \%$ chance of success. During ablation of manifest accessory pathways mapped during sinus rhythm, a short interval between the local ventricular activation and the onset of the QRS was also predictive, as was the presence of a high-frequency deflection between the atrial and ventricular components of the signal suggestive of an accessory pathway potential (Fig. 8). During
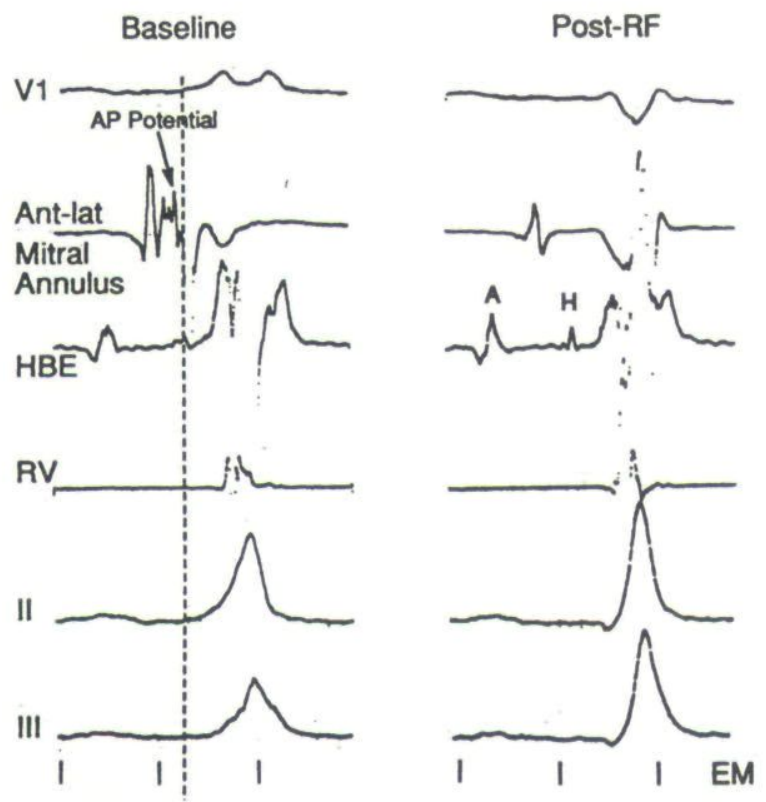

Figure 8. A successful target site in a patient with overt pre-excitation while mapping during normal sinus rhythm. Shown are surface electrocardiographic lead V1, the recording from the distal electrode pair of the ablation catheter located at the anterolateral (Ant-lat) mitral annulus, His-bundle electrogram (HBE), intracardiac recording from the right ventricle $(R V)$, and surface leads II and III. Time lines are $100 \mathrm{msec}$. Note the following features of the ablation site electrogram: (1) the presence of a discrete potential located after the atrial deflection but before the onset of the delta wave (noted by the dotted line), which may represent an accessory pathway (AP) potential; (2) early ventricular activation relative to the onset of the delta wave. This is measured from the peak of the ventricular depolarization in the mapping catheter electrogram to the onset of the delta wave on the surface electrocardiogram and was $-10 \mathrm{msec}$ at this location; and (3) short atrial activation to ventricular activation time. ablation of concealed pathways or pathways mapped during orthodromic reciprocating tachycardia, an accessory pathway potential and the presence of continuous retrograde electrical activity (no isoelectric interval between the ventricular and atrial components of the signal) were significantly correlated with a successful ablation (Fig. 9).

Two large series of patients with accessory pathways treated with radiofrequency catheter ablation have recently been described. ${ }^{14,15}$ Jackman and co-workers ${ }^{15}$ were successful in eliminating accessory pathway conduction in 164 of 166 patients (99\%). Because of recurrence, two sessions were required in 15 patients $(9 \%)$. Complications occurred in only five patients: pericarditis (1), cardiac tamponade (1), complete $\mathrm{AV}$ block (1), and pseudoaneurysm formation at the site of femoral arterial access (2). Calkins and co-workers ${ }^{14}$ were able to eliminate accessory pathway function in 51 of $56(91 \%)$ patients with $4(15 \%)$ requiring two sessions. One patient sustained a myocardial infarction due to inadvertent application of radiofrequency energy within the circumflex coronary artery.

Accessory pathway conduction may sometimes return after initially successful radiofrequency catheter ablation. We recently characterized the incidence and clinical features of accessory pathway recurrence in a series of 130 patients followed for a minimum of 3 months. ${ }^{50}$ Accessory pathway conduction returned in 16 of $130(12 \%)$ patients. Almost half $(7 / 16)$ of these recurrences were within the first 12 hours after ablation and the latest one occurred after 106 days. Return of delta waves on ECG or spontaneous paroxysmal supraventricular tachycardia were the initial indications of recurrence in 15 of 16 patients. Two patients with manifest accessory pathways recurred with exclusively retrograde accessory pathway conduction.

Accessory pathways ablated from the tricuspid annulus (right free wall or septal accessory pathways) had a much higher recurrence rate $(24 \%)$ than those on the mitral annulus $(6 \%)$. Fourteen of 15 patients have had successful repeat accessory pathway ablation after the initial recurrence. Jackman et al. ${ }^{15}$ described recurrence in 15 of $106(9 \%)$ patients with initially successful accessory pathway ablation. The last of these occurred 4.7 months after the procedure. As with our study, there was a disproportionally higher incidence of recurrence among right-sided and septal pathways (14\%) compared with left-sided 

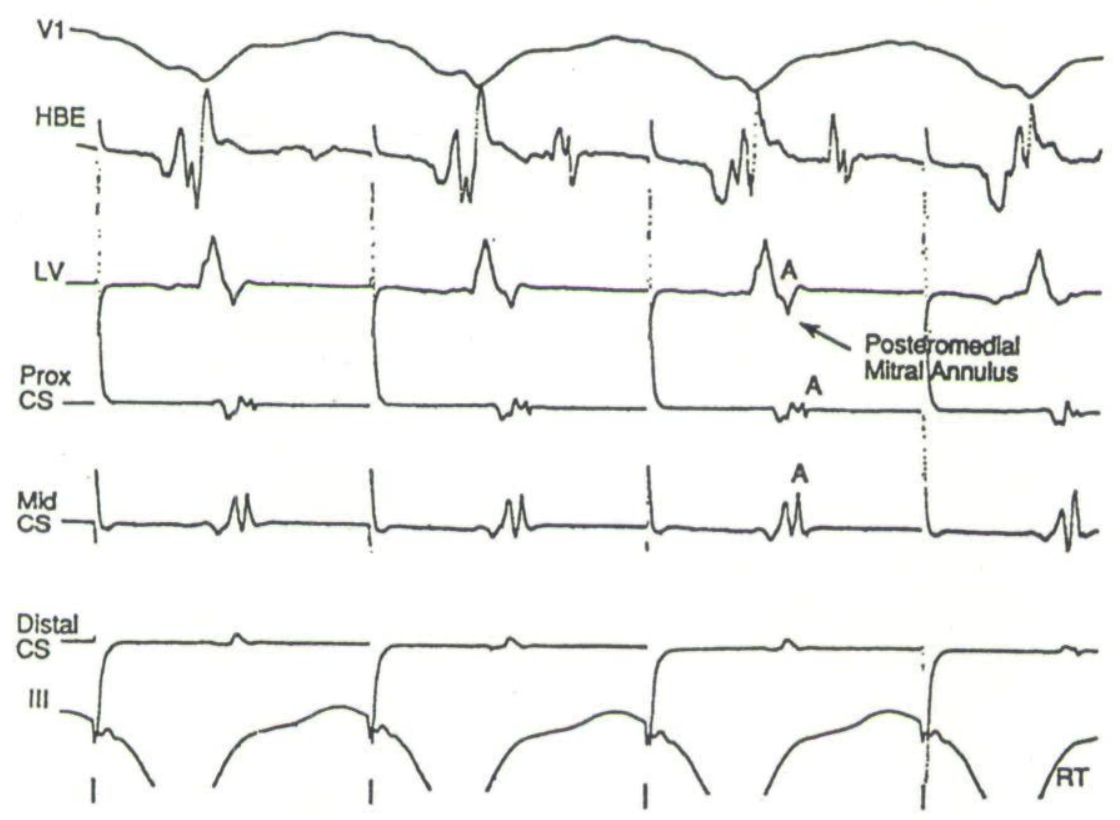

Figure 9. Target site for successful ablation of a concealed left posterior accessory pathway. Shown are the tracings from the electrocardiographic lead V1, His-bundle electrogram (HBE), recordings from the distal electrode pair of the ablation catheter located in the left ventricle $(L V)$ in contact with the posteromedial mitral annulus, recordings from the proximal (Prox), mid, and distal coronary sinus (CS), and surface lead III during ventricular pacing at a cycle length of 500 msec. Note that the ablation site electrogram has: (1) continuous electrical activity from the ventricular to atrial deflection (no intervening isoelectric segment between the ventricular and atrial electrograms in the ablation catheter); and (2) beat-to-beat stability of the electrogram amplitude and morphology. Application of radiofrequency current at 35 watts for 45 seconds resulted in complete loss of accessory pathway conduction.

accessory pathways (5\%). Jackman and coworkers also noted a high efficacy rate of a second ablation session with all 15 of the patients with recurrent accessory pathway function having a successful second session.

\section{Ablation of Ventricular Tachycardia}

The use of radiofrequency current as an energy source for catheter ablation of ventricular tachycardia was first reported in $1987 .{ }^{11}$ In this early report, 3 of 5 patients with ventricular tachycardia were successfully treated with a mean follow-up of 7 months. Subsequent preliminary reports have indicated that radiofrequency current may be used successfully to treat ventricular tachycardia associated with coronary artery disease, arrhythmogenic right ventricular dysplasia, and structurally normal hearts..$^{1-53}$ Another study by Kunze et al. ${ }^{54}$ did not find radiofrequency catheter ablation to be effective in patients with ventricular tachycardia. In this study, radiofrequency ablation was attempted in seven patients with ventricular tachycardia. Six had coronary artery disease and one patient had right ventricular outflow tract tachycardia with a structurally normal heart. Radiofrequency energy was applied at 30 to 40 volts for 10 to 30 seconds without success; follow-up direct current electric shock ablation at the same site was successful in all cases. The relatively low power $(<20 \mathrm{~W})$ used in this study may have contributed to the inefficacy. Because of the variable results in small series of patients, the role of radiofrequency catheter ablation for the treatment of microreentrant ventricular tachycardia remains to be defined.

Radiofrequency catheter ablation appears ideally suited for treatment of ventricular tachycardia due to bundle branch reentry. Bundle branch reentry may be the cause of ventricular tachycardia in $15 \%-30 \%$ of patients with dilated cardiomyopathy from either ischemic or nonischemic causes. ${ }^{55}$ The tachycardia usually has a left bundle branch block configuration and is mediated by antegrade conduction over the right bundle and retrograde conduction via the left bundle. Tchou et al. ${ }^{56}$ showed that this type of ventricular tachycardia could be effectively treated with direct current shock ablation of the 
A $\mathrm{V}_{1}$
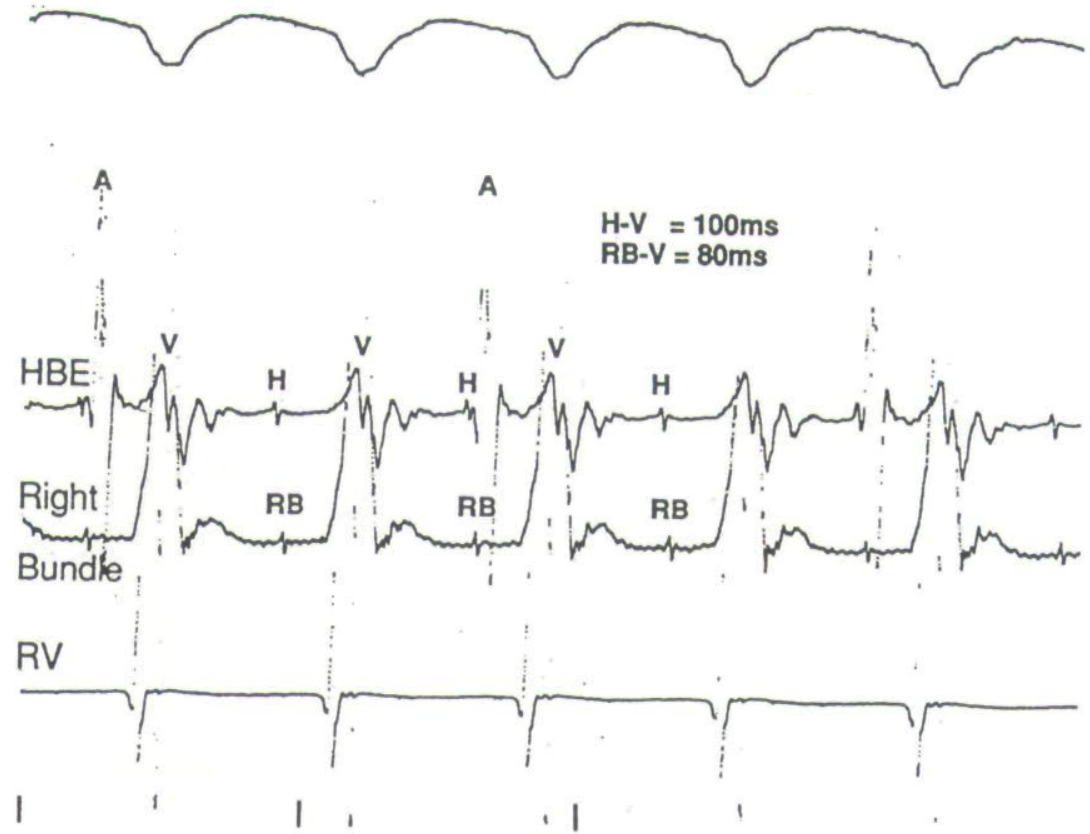

B

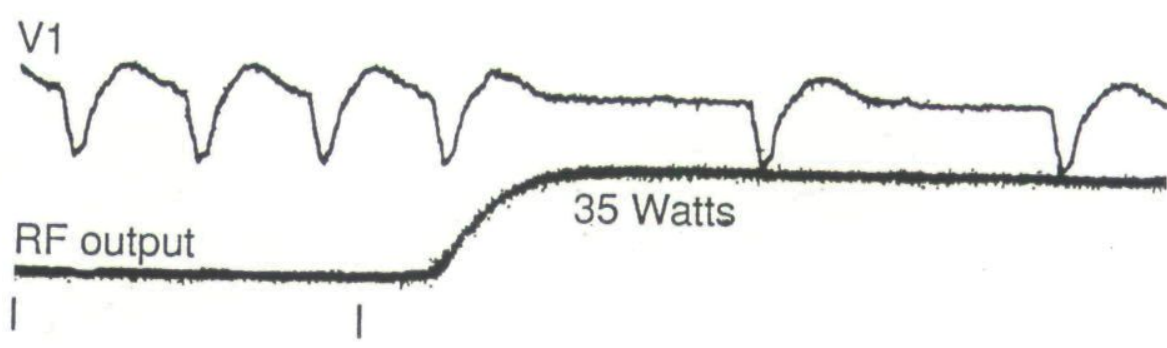

C
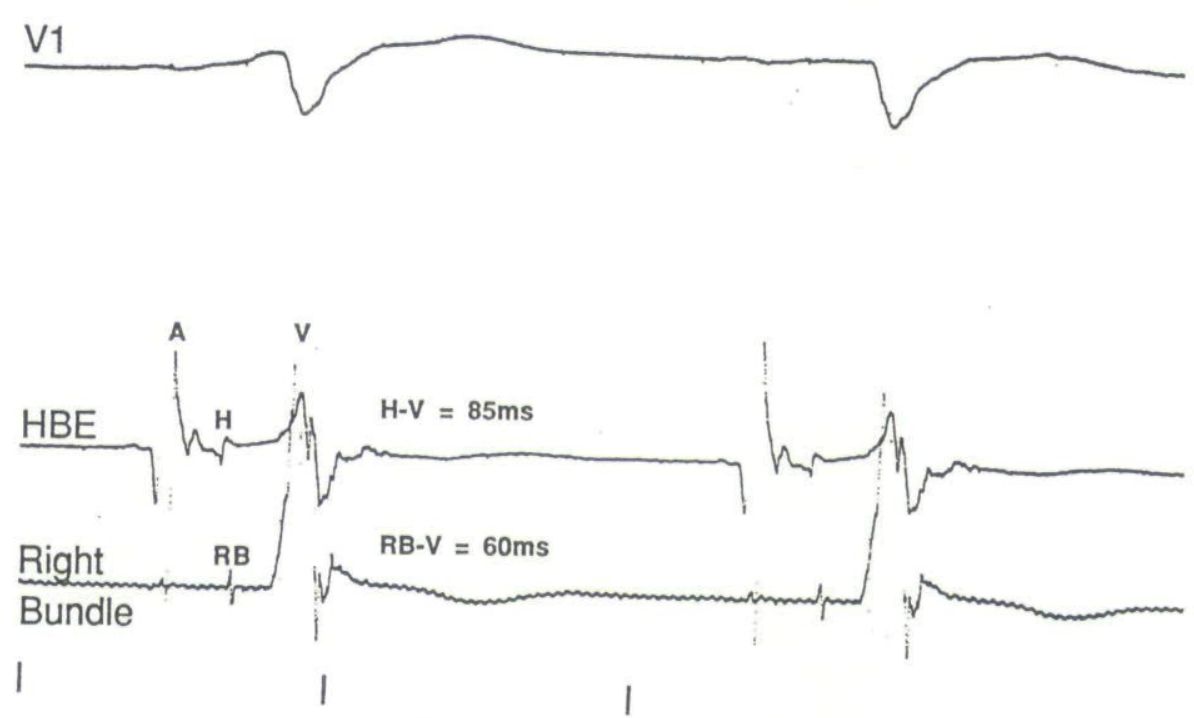

Figure 10. 
D
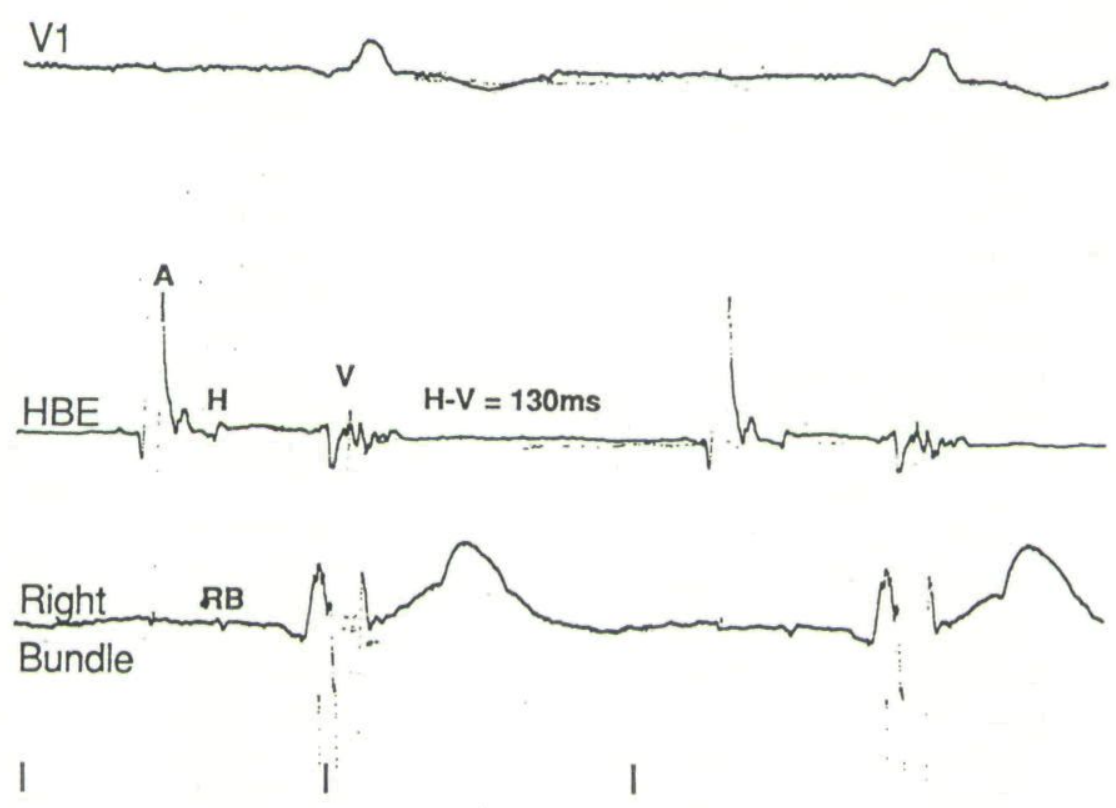

Figure 10. Bundle branch reentrant tachycardia cured by radiofrequency catheter ablation of the right bundle branch. Panel $A$ shows the intracardiac recording obtained during bundle branch reentrant tachycardia. Shown are surface leads VI, His-bundle recording ( $H B E)$, recording from the right bundle branch position, and right ventricular apex (RV). Time lines are $500 \mathrm{msec}$. During the tachycardia there is atrioventricular dissociation as seen in the His-bundle recording. The right bundle deflection $(R B)$ occurs after the His deflection $(H)$, suggesting antegrade conduction over the right bundle. During the tachycardia the $H-V$ interval was $100 \mathrm{~ms}$ and the RB-V interval was $80 \mathrm{~ms}$. Panel B demonstrates immediate termination of the ventricular tachycardia upon application of the radiofrequency current to the right bundle branch. Shown are surface lead $V I$ and the radiofrequency $(R F)$ power output. Time lines are 1 second. The RF current was delivered at a power of 35 watts for 30 seconds to the distal pole of the catheter recording the right bundle branch deflection shown. Panels $C$ and $D$ show the pre- and postablation recordings during normal sinus rhythm. Time lines are 500 ms. Before ablation (C), the surface electrogram has a left bundle branch block morphology, the $H-V$ interval is $85 \mathrm{~ms}$, and the right bundle (RB)-V interval is prolonged to $65 \mathrm{~ms}$. After ablation (D), the surface electrocardiogram demonstrates a right bundle branch block morphology, indicating that the preablation left bundle branch was due to a relative conduction delay in the left bundle. After ablation, the $\mathrm{H}-\mathrm{V}$ interval is increased to $130 \mathrm{~ms}$. The catheter in the right bundle position is still able to record a right bundle branch deflection, suggesting that the site of conduction block in the right bundle is distal to the recording electrodes.

right bundle branch. Radiofrequency energy may also be used to treat bundle branch reentry (Fig. 10). Early case reports have shown that it is a safe and effective means of eliminating conduction over the right bundle and, therefore, eliminating the macroreentrant circuit causing bundle branch reentry. $.57-59$

A preliminary report has also found that radiofrequency energy may be effective in treating idiopathic ventricular tachycardia arising from the right ventricular outflow tract. ${ }^{60}$ In this study, 3 of 3 patients were treated effectively using a mean power output of $36 \mathrm{~W}$. No patient had a recurrence at a follow-up of 1 to 3 months. Idiopathic ventricular tachycardia arising from the left ventricle has also been treated effectively using radiofrequency energy. Two preliminary reports, each with one patient, reported successful ablation after applying radiofrequency current to the site of earliest activation during ventricular tachycardia, which was located in the posteroseptal region in the left ventricle in both cases. ${ }^{58,60}$

\section{Trends for the Future}

Although efficacy rates for radiofrequency catheter ablation of $\mathrm{AV}$ nodal reentry and accessory pathway tachycardias exceeds $90 \%$ at experienced laboratories, procedure durations and fluoroscopy times remain high. Continued 
improvement in the design of ablation catheters promises to shorten and simplify the process. An increasing variety of steerable shaft configurations will undoubtedly facilitate radiofrequency ablation. To have catheters with a range of radii of curvature (as with angiographic catheters) will speed the process of mapping and increase the stability of contact at the ablation site.

Because many variables affect the amount of heating during radiofrequency ablation, energy delivery parameters correlate poorly with lesion volume. The ability to monitor temperature at the electrode-tissue interface using thermometry probes in the distal electrode will provide a more direct indication of the local effects of any given radiofrequency application. This will allow the differentiation between inadequate heating and inadequate positioning as the cause for lack of efficacy of any given radiofrequency application. ${ }^{61}$ The operator will be able to adjust power output to achieve a target temperature, thereby optimizing lesion volume while minimizing the risk of boiling, coagulum formation, and impedance rise. ${ }^{18}$

\section{Regulatory Issues}

Technological and clinical advances in interventional electrophysiology have occurred extremely rapidly. Although radiofrequency catheter ablation is considered the treatment of choice for definitive management of paroxysmal supraventricular tachycardia in selected patients, there is currently no catheter or power supply approved by the Food and Drug Administration for intracardiac use. As a result, the procedure is considered investigational by most institutions. Many insurance carriers, as well as the Health Care Finance Administration, do not reimburse for catheter ablation.

One manufacturer has begun a clinical trial of a radiofrequency ablation system and others will likely follow. Indications for the use of catheter ablation have been published ${ }^{62}$ and guidelines for training personnel and facilities needed to perform ablation are being prepared. These regulatory efforts are of paramount importance to ensure that the rapid dissemination of the technique does not compromise the remarkable track record of safety reported to date.

\section{References}

1. Scheinman MM, Morady F, Hess DS, et al: Catheter-induced ablation of the atrioventricular junction to control refractory supraventricular arrhythmias. J Am Med Assoc 1982;248:851-855.

2. Gallagher JJ, Svenson RH, Kasell JH, et al: Catheter technique for closed-chest ablation of the atrioventricular conduction system. N Engl J Med 1982;306:194-200.

3. Hartzler GO: Electrode catheter ablation of refractory focal ventricular tachycardia. J Am Coll Cardiol 1983;2:1107-1113.

4. Evans GT, Scheinman MM, Zipes DP, et al: The percutaneous cardiac mapping and ablation registry: Final summary of results. PACE 1988; 11:1621-1626.

5. Fox JL: Experimental relationship of radiofrequency electrical current and lesion size for application to percutaneous cordotomy. J Neurosurg 1970; 33:415-421.

6. Organ LW: Electrophysiologic principles of radiofrequency lesion making. Appl Neurophysiol 1976; 39:69-76.

7. Dickson JA, Calderwood SK: Temperature range and selective sensitivity of tumors to hyperthermia: A critical review. Ann NY Acad Sci 1980;335:180205.

8. Franklin JO, Langberg JJ, Oeff M, et al: Catheter ablation of canine myocardium with radiofrequency energy. PACE 1989;12(II):170-181.

9. Huang SK, Jordan N, Graham A, et al: Closed-chest catheter desiccation of atrioventricular junction using radiofrequency energy-A new method of catheter ablation. (Abstract) Circulation 1985;72:III-389.

10. Huang SK, Graham AR, Hoyt RH, et al: Transcatheter desiccation of the canine left ventricle using radiofrequency energy: A pilot study. Am Heart J 1987;1134:43-48.

11. Borggrefe $\mathbf{M}$, Rudde $\mathbf{T}$, Podczeck $\mathbf{A}$, et al: Application of transvenous radio-frequency alternating current ablation in humans. (Abstract) Circulation 1987;76:IV-406.

12. Jackman WM, Wang X, Friday KJ, et al: Catheter ablation of atrioventricular junction using radiofrequency current in 17 patients. Comparison of standard and large-tip catheter electrodes. Circulation 1991;83:1562-1576.

13. Lee MA, Morady F, Kadish A, et al: Catheter modification of the atrioventricular junction with radiofrequency energy for control of atrioventricular nodal reentry tachycardia. Circulation 1991;83:827-835.

14. Calkins H, Sousa J, El-Atassi R, et al: Diagnosis and cure of the Wolff-Parkinson-White syndrome or paroxysmal supraventricular tachycardias during a single electrophysiologic test. N Engl J Med 1991;324:1612-1618.

15. Jackman WM, Wang X, Friday KJ, et al: Catheter ablation of accessory atrioventricular pathways (Wolff-Parkinson-White syndrome) by radiofrequency current. N Engl J Med 1991;324:16051611. 
16. Pearce JA, Geddes LA, Van Vleet JF, et al: Skin burns from electrosurgical current. Med Instrumentation 1983;17(3):225-231.

17. Haines DE, Watson DD: Tissue heating during radiofrequency catheter ablation: A thermodynamic model and observations in isolated perfused and superfused canine right ventricular free wall. PACE 1989;12:962-976.

18. Haines DE: Impedance rise during radiofrequency ablation is due to sudden boiling at the electrode tip and is prevented by tip temperature monitoring. (Abstract) Circulation 1988;78(4):II-156.

19. Wittkampf FHM, Hauer RNW, Robles de Medina EO: Control of radiofrequency lesion size by power regulation. Circulation 1989;80:962-968.

20. Hoyt RH, Huang SK, Marcus FI, et al: Factors influencing trans-catheter radiofrequency ablation of the myocardium. J Appl Cardiol 1986;1:469486.

21. Haverkamp W, Hindricks G, Gulker H, et al: Coagulation of ventricular myocardium using radiofrequency, alternating current: Biophysical aspects and experimental findings. PACE 1989; 12:187-195.

22. Frohmer KJ, Podczeck A, Hief C, et al: Thermal catheter disruption during closed-chest radiofrequency ablation of the atrioventricular conduction system. PACE 1990;13:719-723.

23. An H, Saksena S, Janssen M, et al: Radiofrequency ablation of ventricular myocardium using active fixation and passive contact catheter delivery systems. Am Heart J 1989;118:69-77.

24. Langberg JJ, Lee MA, Chin MC, et al: Radiofrequency catheter ablation: The effect of electrode size on lesion volume in vivo. PACE 1990;13:1242-1248.

25. Wittkampf FH, Kauer RN, Robles de Medina EO: Radiofrequency ablation with a cooled porous electrode catheter. (Abstract) J Am Coll Cardiol 1988;11(2):17A.

26. Huang SKS, Cuaroud H, Tandegueman W: Increase in the lesion size and decrease in the impedance rise with a saline infusion electrode catheter for radiofrequency catheter ablation. (Abstract) Circulation 1989;80(4):II-324.

27. Huang SK, Bharati S, Graham AR, et al: Closed chest catheter desiccation of the atrioventricular junction using radiofrequency energy-A new method of catheter ablation. J Am Coll Cardiol 1987;9:349-358.

28. Borggrefe M, Budde T, Martinez-Rubio A, et al: Radio-frequency catheter ablation for drug-refractory supraventricular tachycardia. (Abstract) Circulation 1988;78:II-305.

29. Langberg JJ, Chin MC, Rosenqvist $\mathbf{M}$, et al: Catheter ablation of the atrioventricular junction with radiofrequency energy. Circulation 1989;80: 1527-1535.

30. Langberg JJ, Chin M, Schamp DJ, et al: Ablation of the atrioventricular junction with radiofrequency energy using a new electrode catheter. Am J Cardiol 1991;67:142-147.

31. Sousa J, El-Atassi R, Rosenheck S, et al: Catheter ablation of the atrioventricular junction from the left ventricle. Circulation 1991;84:567-571.

32. Huang SKS: Predictors of successful catheter ablation of the atrioventricular junction with radiofrequency energy. (Abstract) PACE 1989; 12:675

33. Ross DL, Johnson DC, Denniss R, et al: Curative surgery for atrioventricular junctional ("AV nodal") reentrant tachycardia. J Am Coll Cardiol 1985;6:1383-1392.

34. Cox JL, Holman WL, Cain ME: Cryosurgical treatment of atrioventricular node reentrant tachycardia. Circulation 1987;79:1329-1336.

35. Marcus FI, Blouin LT, Wharton K, et al: Electrophysiological and pathological assessment of chronic first degree atrioventricular block caused by closed-chest catheter ablation with radiofrequency energy in dogs. (Abstract) J Am Coll Cardiol 1987;9(2):95A.

36. Lopez-Merino V, Sanchis J, Chorro FJ, et al: Induction of partial alterations in atrioventricular conduction in dogs by percutaneous emission of high-frequency currents. Am Heart J 1988;115: 1214-1221.

37. Huang SKS, Bharati S, Graham AR, et al: Chronic incomplete atrioventricular block induced by radiofrequency catheter ablation. Circulation 1989; 80:951-961.

38. Goy JJ, Fromer M, Schlaepfer J, et al: Clinical efficacy of radiofrequency current in the treatment of patients with atrioventricular node reentrant tachycardia. J Am Coll Cardiol 1990;16:418-423.

39. Sanjuan R, Morell S, Civera RG, et al: Transvenous ablation with high frequency energy for atrioventricular junctional (AV nodal) reentrant tachycardia. PACE 1989;12:1631-1639.

40. Huang SKS, Chenarides J, Gasdia G: Ablation of the dual pathways and retrograde conduction in patients with atrioventricular nodal reentrant tachycardia by radiofrequency catheter ablation. (Abstract) Circulation 1989;80:II-41 .

41. Kunze K-P, Schluter M, Kuck K-H: Radiofrequency modulation of AV nodal conduction for treatment of AV nodal tachycardia. (Abstract) Circulation 1989;80:II-324.

42. Mitrani R, Miles WM, Klein LS, et al: Atrioventricular nodal reentrant tachycardia modification by radiofrequency catheter ablation: Mechanism of successful ablation. (Abstract) J Am Coll Cardiol 1991;17:323A.

43. Mletzko R, Jung W, Manz M, et al: Radiofrequency catheter ablation of retrograde pathways in patients with AV nodal reentry tachycardia. (Abstract) J Am Coll Cardiol 1991;17:366A.

44. Roman CA, Wang X, Friday KJ, et al: Catheter technique for selective ablation of slow pathway in AV nodal reentrant tachycardia. (Abstract) PACE 
$1990 ; 13: 498$.

45. Langberg J, Kou W, Calkins $\mathrm{H}$, et al: Conversion from typical to "atypical" AV nodal reentry tachycardia (AVNRT) after radiofrequency catheter modification of the AV junction. (Abstract) J Am Coll Cardiol 1991;17:337A.

46. Swartz J, Tracy C, Fletcher R, et al: A comparative study of direct current and radiofrequency atrial endocardial ablation of accessory pathways. (Abstract) J Am Coll Cardiol 1991;17:109A.

47. Wang X, Moulton K, Beckman K, et al: Optimal electrode site for radiofrequency ablation of posteroseptal accessory AV pathways. (Abstract) J Am Coll Cardiol 1991;17:232A.

48. Wang X, Moulton K, Margolis D, et al: Pulse duration required for radiofrequency catheter ablation of accessory pathways. (Abstract) Circulation 1990;82:III-719.

49. Calkins H, Kim YN, Schmaltz S, et al: Electrogram criteria for identification of appropriate target sites for radiofrequency catheter ablation of accessory atrioventricular connections. Circulation 1992; (In press).

50. Langberg JJ, Calkins $\mathrm{H}, \mathrm{Kim} \mathrm{YN}$, et al: Recurrence of conduction in accessory atrioventricular connections after initially successful radiofrequency catheter ablation. (Abstract) Circulation 1992; (In press).

51. Borggrefe $\mathbf{M}$, Hief $\mathbf{C}$, Karbenn $\mathrm{U}$, et al: Transcatheter ablation of ventricular tachycardia in patients with and without coronary artery disease (CAD). (Abstract) Circulation 1990;82:III-550.

52. Borggrefe $\mathbf{M}$, Wichter $\mathrm{T}$, Karbenn $\mathrm{U}$, et al: Catheter ablation of drug-refractory ventricular tachycardia in patients with arrhythmogenic right ventricular disease. (Abstract) Circulation 1990;82: III-167.

53. Davis MJE, Murdock C: Radiofrequency catheter ablation of refractory ventricular tachycardia. PACE 1988;11:725-729.
54. Kunze K-P, Kuck K-H, Schluter M: Radiofrequency or direct current for ablation of ventricular tachycardia? (Abstract) J Am Coll Cardiol 1989;13:176A.

55. Caceres J, Jazayeri M, McKinnie J, et al: Sustained bundle branch reentry as a mechanism of clinical tachycardia. Circulation 1989;79:256-270.

56. Tchou P, Jazayeri M, Denker S, et al: Transcatheter electrical ablation of the right bundle branch: A method of treating macro-reentrant ventricular tachycardia due to bundle branch reentry. Circulation 1988;78:246-257.

57. Langberg JJ, Desai J, Dullet N, et al: Treatment of macroreentrant ventricular tachycardia with radiofrequency ablation of the right bundle branch. Am J Cardiol 1989;63:1010-1013.

58. Bowman A, Fitzgerald D, Friday K, et al: Catheter ablation of selected segments of the AV conduction system using radiofrequency current. (Abstract) PACE 1988;11:489.

59. Cohen TJ, Chien WW, Lurie KG, et al: Radiofrequency catheter ablation for bundle branch reentrant tachycardia. (Abstract) PACE 1991; 14:682.

60. Klein LS, Miles WM, Gering LE, et al: Radiofrequency catheter ablation of ventricular tachycardia in patients without structural heart disease. (Abstract) J Am Coll Cardiol 1991; 17:91A.

61. Hindricks G, Haverkamp W, Rissel U, et al: Catheter tip temperature as a determinant of lesion size during radio-frequency coagulation in situ. (Abstract) Eur Heart J 1988;9:263.

62. Scheinman MM, Laks MM, DiMarco J, et al: Current role of catheter ablative procedures in patients with cardiac arrhythmias. A report for health professionals from the subcommittee on electrocardiography and electrophysiology, American Heart Association. Circulation 1991;83:21462153. 
This document is a scanned copy of a printed document. No warranty is given about the accuracy of the copy. Users should refer to the original published version of the material. 